\title{
Fenología reproductiva y productividad de Oenocarpus bataua (Mart.) en bosques inundables del Chocó Biogeográfico, Colombia
}

\author{
Lucas Cifuentes ${ }^{1,4}$, Flavio Moreno ${ }^{2} \&$ Diego Andrés Arango ${ }^{3}$ \\ ${ }^{1}$ Maestría en Bosques y Conservación Ambiental, Universidad Nacional de Colombia, Sede Medellín, \\ calle 59a, n. 63-020, Autopista Norte, bloque 20, piso 2, oficina 20-204, A.A. 1027, Medellín, Colombia \\ ${ }^{2}$ Grupo de Investigación en Ecología y Silvicultura de Especies Forestales Tropicales, \\ Departamento de Ciencias Forestales, Universidad Nacional de Colombia, Sede Medellín, \\ calle 59a, n. 63-020, Autopista Norte, bloque 14, piso 4, oficina 14-422, A.A. 1027, Medellín, Colombia \\ ${ }^{3}$ Doctorado en Ecología, Universidad Nacional de Colombia, Sede Medellín, calle 59a, n. 63-020, \\ Autopista Norte, bloque 20, piso 2, oficina 20-204, A.A. 1027, Medellín, Colombia \\ ${ }^{4}$ Autor para correspondencia: Lucas Cifuentes Gómez, e-mail: lcifuen@unal.edu.co
}

CIFUENTES, L., MORENO, F. \& ARANGO, D.A. Reproductive phenology and fruit productivity of Oenocarpus bataua (Mart.) in flooded forests in the Chocó Biogeographic region, Colombia. Biota Neotrop. 10(4): http://www.biotaneotropica.org.br/v10n4/en/abstract?article+bn01910042010.

\begin{abstract}
Oenocarpus bataua (Mart.) is a palm species forming highly dense stands in flooded forests of the Chocó Biogeographic region. This species is considered promising because its fruits contain protein and high quality oil; therefore, periodic harvests of fruits are a potential source of food and income to local communities and could contribute to improve their living conditions. We studied the reproductive phenology and productivity of fruits of this species in two forest types: mono-dominant stands, called locally "pure milpesal"-MP (occasionally flooded), and more heterogeneous forests, called "mixed murrapal"-MM (periodically flooded), with specific densities of 278 and 82 individuals.ha ${ }^{-1}$, respectively. In each forest type, we established a block with 12 plots of $20 \times 25 \mathrm{~m}$, where the reproductive palms were labeled (166 in MP and 49 in MM). Every other week, from November 1999 until December 2001 (26 months) and from November 2006 until May 2009 (31 months), the phenological state of each previously labeled palm was recorded. The phenological behavior was quantified using the Activity Index and the observed proportions of each phenophase along time were evaluated with different regression models. According to these models, a phenological cycle of the species at the population level lasts about 4 years. We could not find any relationship between rainfall and phenological variables; therefore, the phenological cycle appears to respond to endogenous factors. The production peak of ripe fruits in the population occurred every 34 months in 47 and 26\% in all palms in MP and MM. Fruit productivity per cycle was 3014 and $330 \mathrm{~kg} \cdot \mathrm{ha}^{-1}$, respectively.
\end{abstract}

Keywords: palm tree, flowering, fruiting, synchrony, reproductive cycle.

CIFUENTES, L., MORENO, F. \& ARANGO, D.A. Fenología reproductiva y productividad de Oenocarpus bataua (Mart.) en bosques inundables del Chocó Biogeográfico, Colombia. Biota Neotrop. 10(4): http://www. biotaneotropica.org.br/v10n4/es/abstract?article+bn01910042010.

Resumen: Oenocarpus bataua (Mart.) es una especie de palma que conforma rodales altamente densos en bosques inundables del Chocó Biogeográfico. Es considerada promisoria porque sus frutos contienen proteínas y aceite de alta calidad; por ello, el manejo sostenible de estos bosques y la cosecha periódica de sus frutos pueden proveer alimento e ingresos monetarios a las comunidades locales y, así, mejorar su calidad de vida. Se estudió la fenología reproductiva y la productividad de frutos de esta especie en dos comunidades vegetales: milpesal puro-MP (inundable esporádicamente) y murrapal mezclado-MM (inundable periódicamente), con densidades específicas de 278 y 82 individuos.ha ${ }^{-1}$, respectivamente. En cada comunidad se estableció un bloque con 12 parcelas de $20 \times 25 \mathrm{~m}$, donde se marcaron las palmas reproductivas (166 en MP y 49 en MM). Quincenalmente, entre noviembre de 1999 y diciembre de 2001 (26 meses), y entre noviembre de 2006 y mayo de 2009 (31 meses), se registró el estado fenológico de cada palma. Los patrones reproductivos fueron cuantificados mediante el índice de actividad y las proporciones de ocurrencia de cada fenofase fueron evaluadas mediante diferentes modelos de regresión. De acuerdo con éstos, un ciclo fenológico de la especie a nivel poblacional, desde la aparición del botón floral y posterior exposición de las inflorescencias, hasta el desarrollo, maduración y caída de los frutos dura $c a$. 4 años. No se encontró una relación significativa entre la precipitación y la ocurrencia de las fenofases, por lo que su ciclo fenológico parece responder a factores endógenos. El pico de producción de frutos maduros en la población ocurrió cada 34 meses y se presentó en 47 y $26 \%$ de las palmas en MP y MM. La productividad de frutos por ciclo fue 3014 y $330 \mathrm{~kg} \cdot \mathrm{ha}^{-1}$, respectivamente.

Palabras-clave: palma arbórea, floración, fructificación, sincronía, ciclo reproductivo. 


\section{Introducción}

O. bataua es considerada como una especie promisoria, tanto por sus frutos ricos en aceite de excelente condición, similar al aceite de oliva y con proteínas de alta calidad, como por su especial adaptación a suelos pobres (Galeano \& Bernal 1987, Balick 1992, Morcote-Ríos et al. 1998). Es ampliamente usada en la cuenca amazónica y sus frutos son especialmente importantes en la dieta de poblaciones indígenas (Miller 2002). En la cuenca del Río Atrato (Chocó Biogeográfico), $O$. bataua es una palma dominante en los bosques inundables, y forma densas y extensas poblaciones; los habitantes de la región reconocen la utilidad de la palma y, ocasionalmente, utilizan sus frutos maduros para preparar bebidas o extraer aceite.

Debido a su importancia como fuente alimenticia para el hombre y la fauna silvestre, así como su potencial económico, se han desarrollado varios estudios para establecer los patrones de la fenología reproductiva y la productividad de frutos. En el Pacífico colombiano, Collazos \& Mejía (1988) relacionaron la aparición de frutos maduros con el inicio de la época húmeda; estos autores plantearon la existencia de una producción individual alternada: alta en un año y baja o nula al siguiente, con un promedio anual de $236 \mathrm{~kg} \cdot \mathrm{ha}^{-1}$. En el occidente de la Amazonía, la floración de $O$. bataua presentó un patrón continuo y se relacionó principalmente con el fotoperíodo (Stevenson et al. 2008), con una productividad de frutos maduros hasta de 262,1 kg/ha/año (Stevenson 2004). En Ecuador, Miller (2002) notó que el ciclo fenológico de la especie, a nivel poblacional, fue bianual; frutos verdes y maduros se presentaron durante todo el año y el pico de producción de frutos maduros ocurrió cada 2 años, al final de la estación seca e inicio de la estación húmeda, con una producción media de $700 \mathrm{~kg} \cdot \mathrm{ha}^{-1}$.

Ruiz \& Alencar (2004) estudiaron la fenología reproductiva de $O$. bataua en Brasil durante 16 años, al igual que su relación con variables meteorológicas. Estos autores encontraron que las fenofases ocurrían durante todo el año y que la producción de frutos maduros se correlacionó significativamente con la insolación, temperatura y precipitación; sin embargo, no incluyeron análisis sobre sincronía y ciclos reproductivos. Más recientemente, en un bosque de montaña de los Andes colombianos, se realizó un análisis del comportamiento fenológico, a nivel individual y poblacional, durante 4 años (RojasRobles \& Stiles 2009). Allí se encontró que un evento reproductivo exitoso en un individuo requirió, en promedio, ca. 2 años desde la aparición del botón floral hasta el racimo seco. A nivel poblacional, es decir, cuando se considera el comportamiento fenológico de todos los individuos evaluados, en conjunto, el tiempo transcurrido entre dos picos de floración fue ca. 4 años. La sincronía individual y poblacional fue baja.

Los resultados de estos estudios, los cuales se desarrollaron en distintas regiones geográficas, presentan diferencias en la sincronía y proporción de ocurrencia de los eventos fenológicos, así como en la duración, regularidad y estacionalidad del ciclo fenológico, y en la productividad de frutos de O. bataua. Se ha encontrado que la fenología de muchas especies es controlada principalmente por factores ambientales (Borchert 1994, Bach 2002, Schöngart et al. 2002, Wallace \& Painter 2002, Borchert et al. 2004, Do et al. 2005, Haugaasen \& Peres 2005, McLaren \& McDonald 2005) y quizás, por esa causa, muchas especies tropicales de amplia distribución difieren en su comportamiento fenológico entre regiones o ecosistemas (Borchert 1994, Newstrom et al. 1994, Bencke \& Morellato 2002a, Williams-Linera 2003). De acuerdo con estos antecedentes, nos planteamos la hipótesis de que los patrones fenológicos de $O$. bataua son diferentes para cada región y entre ecosistemas, pues están asociados con factores ambientales.
En esta investigación se quiere responder las siguientes preguntas: 1) Existen diferencias en la fenología reproductiva de $O$. bataua entre dos ecosistemas diferentes? 2) Se aprecia alguna relación entre los patrones fenológicos y la precipitación? 3) Los eventos fenológicos ocurren de forma sincrónica entre los individuos de la población? 4) Cuál es la duración del ciclo reproductivo de las poblaciones bajo estudio? 5) Cuál es el tiempo medio de desarrollo de cada estructura reproductiva en un individuo? 6) Se presentan diferencias en el tiempo de desarrollo de las estructuras reproductivas entre ecosistemas y períodos de observación? 7) Cuál es la productividad de frutos maduros en los bosques estudiados?

Responder las preguntas anteriores nos permitirá alcanzar los objetivos principales de este estudio, los cuales son: 1) describir el comportamiento de la fenología reproductiva de $O$. bataua en la cuenca media del río Atrato, Colombia; y 2) determinar la productividad de frutos maduros de la especie en los bosques de la región. Este conocimiento es fundamental para el manejo sostenible de $O$. bataua en la zona, ya que las cosechas periódicas de frutos pueden generar alimento e ingresos monetarios a las comunidades locales, y mejorar así sus condiciones de vida.

\section{Materiales y Métodos}

\section{1. Área de estudio}

Este trabajo se llevó a cabo en la parte media de la llanura de inundación del Río Atrato, Colombia, entre los municipios de Vigía del Fuerte (Antioquia) y Bojayá (Chocó); región que pertenece a la provincia del Chocó Biogeográfico y cuya altitud promedia es 100 msnm. Los numerosos afluentes del Río Atrato, sumados a la alta precipitación y a las características del relieve, generan condiciones de inundabilidad en los suelos cercanos a caños y ríos, por lo que los diferentes tipos de bosque se distribuyen de acuerdo con las características de inundación.

Basados en el diagnóstico sobre el componente arbóreo y el mapa de asociaciones vegetales desarrollado por la Universidad Nacional de Colombia (1996) para la zona, se eligieron dos ecosistemas donde esta especie tiene una presencia importante: 1) Milpesal Puro (MP, $6^{\circ} 37^{\prime} 11^{\prime \prime} \mathrm{N} \mathrm{y} 76^{\circ} 54^{\prime} 05^{\prime \prime} \mathrm{W}$ ), dominado por $O$. bataua y con registros significativos de Campnosperma panamense (Standl.); los suelos de esta asociación vegetal son poco afectados por las inundaciones. 2) Murrapal Mezclado (MM, 6 34' 50" N - 76 52'09' W), dominado moderadamente por Euterpe oleracea (Mart.) y con presencia de O. bataua, Manicaria saccifera (Gaertn.) y Swartzia sp., entre otras; sus suelos están sometidos a inundaciones periódicas.

De acuerdo con la clasificación ecológica de Holdridge (1978), el área corresponde a la zona de vida bosque muy húmedo tropical (bmh-T). Allí se presenta una temperatura media anual de $28{ }^{\circ} \mathrm{C}$, humedad relativa de $89 \%$ y precipitación media anual entre 5000 y $7000 \mathrm{~mm}$; esta última exhibe una distribución bimodal a lo largo del año, caracterizada por la presencia alternada de dos temporadas muy lluviosas y dos relativamente menos lluviosas. La época más seca tiene lugar entre diciembre y marzo, con precipitaciones mínimas mensuales superiores a $200 \mathrm{~mm}$, en promedio (Eslava 1993). Los suelos de ambos ecosistemas poseen fertilidad baja, su origen es aluvial y están sometidos a inundaciones frecuentes cuya intensidad depende de la posición fisiográfica (Cortés 1993).

\section{Especie bajo estudio}

Oenocarpus bataua (Mart.) es una de las especies con mayor presencia en los bosques neotropicales (Galeano \& Bernal 1987, Kahn 1991, Balick 1992, Henderson et al. 1995). Es una palma arbórea de estipe solitario, liso y conspicuamente anillado; alcanza 
alturas hasta de $25 \mathrm{~m}$. Es monoica, con inflorescencias infrafoliares; cada inflorescencia puede alcanzar hasta $2 \mathrm{~m}$ de largo, incluyendo pedúnculos florales hasta de $40 \mathrm{~cm}$ (Núñez-Avellaneda \& RojasRobles 2008). Los frutos inmaduros son de color verde y al madurar se tornan morados a negros (Balick 1992).

\section{Trabajo de campo}

El seguimiento fenológico de la especie se abordó en MP y MM. En cada asociación vegetal se estableció un bloque rectangular de $100 \times 210 \mathrm{~m}$, conformado por 12 parcelas de $20 \times 25 \mathrm{~m}\left(500 \mathrm{~m}^{2}\right)$ dispuestas en dos hileras paralelas de 6 y separadas entre sí por corredores de $10 \mathrm{~m}$ de ancho; en estas parcelas se adelantaron también otros estudios de dinámica del ecosistema. Se marcaron todas las palmas adultas con evidencias de estructuras reproductivas: 166 en MP y 49 en MM. Los eventos fenológicos se registraron cada 15 días durante dos períodos: I, entre noviembre de 1999 y diciembre de 2001 (26 meses), y II, entre noviembre de 2006 y mayo de 2009 (31 meses). Estos eventos, observados directamente en los individuos marcados con el apoyo de binoculares, se clasificaron así: 1) botón floral o bráctea peduncular leñosa que envuelve la inflorescencia; 2) inflorescencia sin fecundar, cuando se abre el botón floral y se expone la inflorescencia hasta que la flores femeninas se fecundan o caen; 3) inflorescencia fecundada, cuando las flores femeninas son fecundadas hasta que se pueden ver los frutos en formación; 4) infrutescencia con frutos inmaduros, desde que se desarrollan los frutos hasta que cambian de color verde a morado; 5) infrutescencia con frutos maduros, cuando los frutos cambian de color verde a morado hasta que caen o son consumidos; y 6) racimos secos, cuando todos los frutos de la infrutescencia han caído completamente.

\section{Análisis de datos}

La sincronía intraespecífica, entendida como la ocurrencia simultánea del mismo evento fenológico en los individuos muestreados de la población (Newstrom et al. 1994), se evaluó en ambos ecosistemas mediante el índice de actividad. Este método de análisis es de carácter cuantitativo a nivel poblacional, ya que indica la proporción de individuos observados que manifiesta determinado evento fenológico (Bencke \& Morellato 2002a); así, a mayor proporción de individuos que presentan simultáneamente una fenofase, mayor es la sincronía de la población. Un evento fenológico fue considerado como no sincrónico o asincrónico cuando menos de $20 \%$ de los individuos presentó la fenofase, poco sincrónico o con sincronía baja cuando la fenofase ocurrió entre 21 y $60 \%$ de los individuos, y con sincronía alta cuando más de $60 \%$ de los individuos exhibió la fenofase (Bencke \& Morellato 2002b). La definición de sincronía se planteó para botón floral, frutos verdes (flores fecundadas y frutos inmaduros conjuntamente) y frutos maduros.

Para establecer la similitud del ciclo fenológico reproductivo de $O$. bataua en ambos tipos de bosque, se calcularon coeficientes de correlación de Pearson entre la proporción mensual de individuos que presentó cada fenofase en cada ecosistema, con desfases en el tiempo (time lag) hasta de 10 meses. Con el propósito de describir la variación temporal y duración de cada fenofase, así como del ciclo fenológico a nivel poblacional, la proporción de individuos que presentó cada fenofase, obtenida a partir del índice de actividad, se ajustó mediante diferentes modelos. La proporción de individuos con frutos verdes se modeló utilizando una función sinusoidal de la forma $Y=A \cos (k t+\varphi)$, donde: $Y$-proporción de individuos con frutos verdes, $A$-amplitud de la función, $k=2 \pi / T$ ( $T$, período de la función), $t$-tiempo (mes), y $\varphi$-desfase de la función. Esta función permitió definir el patrón cíclico de la producción de frutos verdes de la población. Para los casos de botón floral y fruto maduro, los mejores ajustes se obtuvieron con modelos de regresión potencial.
Antes de modelar la función sinusoidal, puesto que la función coseno varía entre -1 y 1 , fue necesario transformar los valores observados de proporción de individuos con frutos verdes $(Y(t))$ a un índice que oscile entre tales valores, el cual se obtuvo como la diferencia entre la proporción observada en cada tiempo $(t)$ y el promedio general de las proporciones. De esta forma, con la variable transformada, se ajustó el modelo sinusoidal. Luego, los valores modelados (en torno a 0 ) se transformaron nuevamente a proporción de individuos con frutos verdes, al sumar a cada valor el promedio de las proporciones.

La duración de las estructuras reproductivas individuales se determinó mediante la diferencia de días entre la aparición y desaparición de cada una de ellas. Para esto se utilizó la información de todas las estructuras que alcanzaron su desarrollo con éxito. Posteriormente, a partir de los datos individuales, se calcularon tiempo medio, desviación estándar, coeficiente de variación e intervalos de confianza de la duración de cada una de ellas. Las comparaciones entre ecosistemas, en cada período de observación de esta variable, se realizaron mediante análisis de varianza de una vía. En el período I no se hizo la comparación del tiempo de permanencia de frutos maduros entre ecosistemas debido a que en MM no se registró esta fenofase en ninguna palma; por lo tanto, la comparación de frutos maduros entre ecosistemas sólo se hizo con la información obtenida para el período II.

Las relaciones entre precipitación mensual y la proporción media mensual de individuos con botones florales, frutos verdes o frutos maduros en ambos ecosistemas, se determinaron mediante el coeficiente de correlación de Pearson; además, se evaluaron las relaciones entre cada evento fenológico y la precipitación hasta de 10 meses atrás (time lag). Se ha encontrado que los eventos fenológicos se asocian más con la disponibilidad de agua en el suelo que con la precipitación (Borchert 1994, 1999, Schöngart et al. 2002, Borchert et al. 2004); en estos bosques inundables dicha disponibilidad depende del río Atrato. Desafortunadamente, los datos sobre el nivel del río en la zona de estudio son escasos y se restringen al primer período de registros fenológicos (años 1999 - 2001); por lo anterior, se utilizó la precipitación mensual como variable sustituta de la disponibilidad de agua en el suelo, ya que la relación entre ésta y nivel medio del río fue significativa para ese período (coeficiente de correlación de Pearson =0,76; $\mathrm{p}=0,0000$ ). Al hacer lo mismo con los datos climáticos históricos, para un período de 15 años (1985-2000), encontramos una correlación un poco menor pero aún significativa $(\mathrm{r}=0,64 ; \mathrm{p}<0,0000)$, lo cual sugiere que la precipitación local ejerce una influencia moderadamente alta sobre los niveles del río en el área de estudio.

\section{Productividad}

Mediante recorridos de campo, se identificaron y cortaron 30 racimos con frutos maduros en MP; aquí se utilizó un gancho tipo malayo, extensible hasta $15 \mathrm{~m}$ de longitud. El gancho malayo consiste en un tubo desplegable en cuyo extremo se encuentra fija una cuchilla en forma de media luna; ésta se engancha en el racimo para hacer el corte. Los racimos cortados cayeron dentro de una malla previamente ubicada en la base del estipe de la palma. Cada racimo cortado se desgranó y los frutos se pesaron en una balanza con capacidad de $25 \mathrm{~kg}$ ( $\pm 200 \mathrm{~g}$ ). El número total de frutos por racimo se estimó a partir del peso de una muestra de 100 frutos tomada de cada racimo. Finalmente, para ambas asociaciones vegetales, se calculó el porcentaje de palmas que produjeron racimos con frutos maduros, el número total de racimos y el peso total de frutos por hectárea por ciclo productivo. 


\section{Resultados}

\section{Ciclo reproductivo de la población}

Se presentó alta correlación lineal de los valores mensuales de la proporción de individuos que exhibió cada fenofase entre MP y MM en los dos períodos evaluados (Tabla 1); esto sugiere la similitud en la regularidad del ciclo reproductivo de la especie entre ambos ecosistemas.

La ocurrencia de la fenofase botón floral se ajustó a un modelo de regresión potencial de orden $3(B F=-0,901944+0,147176 t+$ $\left.0,008682 t^{2}+0,000128 t^{3}, \mathrm{R}^{2}=94,89 \%, \mathrm{p}=0,0000\right)$ y su duración se estimó en 28 meses. La función sinusoidal modelada para la ocurrencia de la fenofase fruto verde $(Y=0,262340 \cos (0,186079 t$ $\left.-2,768492), \mathrm{R}^{2}=95,59 \%, \mathrm{p}=0,0000\right)$ demostró su comportamiento cíclico; con base en el mismo modelo, se calculó la duración del ciclo de producción de frutos verdes a nivel poblacional en 34 meses. En cuanto a la fenofase fruto maduro, ésta se ajustó a un modelo de regresión potencial de orden $4(F M=0,059601-0,071716 t+$ $\left.0,022413 t^{2}-0,001742 t^{3}+0,000039 t^{4}, \mathrm{R}^{2}=94,52 \%, \mathrm{p}=0,0000\right)$ y su duración en la población se estimó en ca. 22 meses. Los modelos de estas fenofases se ilustran en las Figuras 1a, b y c, respectivamente, y las duraciones de cada una de ellas en la Figura 1d.

De acuerdo con estos modelos, el ciclo reproductivo completo de la especie a nivel poblacional dura 48 meses. No obstante, existe un traslape de ciclos, de manera que un nuevo ciclo reproductivo inicia a los 34 meses de haber comenzado el ciclo anterior. La producción de frutos verdes inició 12 meses después del comienzo de la producción de botones florales y alcanzó sus valores máximos a los 28-30 meses después de iniciado el ciclo completo. Este pico coincidió con el final de la fenofase botón floral y la aparición de frutos maduros. Finalmente, los frutos maduros desaparecieron a los 48 meses, poco después del final de la fenofase fruto verde (Figura 1d).

\section{Sincronía de fenofases}

A partir del índice de actividad y con la ayuda de cada modelo seleccionado, se evaluó la sincronía de cada fenofase a nivel poblacional. Así, la fenofase botón floral fue poco sincrónica, dado que la proporción de palmas que presentó el evento fue inferior a $60 \%$ durante la mayor parte del tiempo, excepto en los meses siete y ocho desde el inicio de la fenofase, cuando ocurrieron los máximos valores de ocurrencia (cercana a 70\%) y, por tanto, una sincronía relativamente alta (Figuras 1a, d). La fenofase fruto verde también fue poco sincrónica la mayor parte del tiempo; su presencia máxima ocurrió durante los meses 16 y 17 después de iniciado el evento, cuando alcanzó una proporción superior a $60 \%$ (Figuras $1 \mathrm{~b}, \mathrm{~d}$ ). La fenofase fruto maduro se comportó de forma asincrónica, ya que se presentó en menos de $20 \%$ de los individuos durante casi la totalidad del tiempo de registro. El pico máximo de ocurrencia de frutos maduros ocurrió entre los meses 9 y 11 posteriores al inicio de la fenofase, cuando alcanzó valores por encima de $20 \%$ (Figuras 1c, d).

\section{Duración de estructuras reproductivas}

Cada estructura reproductiva tuvo una duración variable. El valor medio de la fenofase botón floral, desde su aparición hasta que se rompe la bráctea y expone las flores, fue 6,27 meses $(\mathrm{DE}=2,52)$; para la fenofase flor ( $\sin$ fecundar), el lapso fue 0,80 meses ( $D E=0,35$ ). El racimo de frutos verdes tuvo una duración media de 11,56 meses $(\mathrm{DE}=2,94)$, mientras que el valor medio para frutos maduros fue 5,18 meses $(\mathrm{DE}=1,75)$ (Tabla 2). El tiempo transcurrido entre la aparición del botón floral hasta la maduración y caída de frutos fue, en promedio, 23,8 meses. La duración de las estructuras reproductivas en cada período de observación no presentó diferencias significativas entre ecosistemas (Tabla 3).

\section{Relación fenología - clima}

No se encontró relación significativa alguna entre precipitación y cada una de las fenofases evaluadas; tampoco ocurrió con la precipitación mensual retrasada hasta 10 meses. En la Tabla 4 se presentan los valores del coeficiente de correlación de Pearson evaluados entre cada fenofase y la precipitación hasta de los 4 meses anteriores.

\section{Productividad}

No todos los botones florales se convirtieron en frutos maduros. En MP, 74\% de éstos se convirtió en racimos de frutos verdes y sólo $24 \%$ llegó al estado de fruto maduro (33\% de frutos verdes); en

Tabla 1. Correlación de los valores mensuales de las fenofases entre ecosistemas de milpesal puro y murrapal mezclado para cada período evaluado (valor superior: coeficiente de correlación de Pearson, valor inferior entre paréntesis: significancia estadística).

Table 1. Correlation of monthly values of phenophases between ecosystems of pure milpesal and mixed murrapal for each period (top of each cell: Pearson's correlation coefficient, bottom in parenthesis: $\mathrm{P}$ value)

\begin{tabular}{|c|c|c|c|c|c|c|}
\hline \multirow[t]{2}{*}{ Fenofase } & \multicolumn{3}{|c|}{ Período I } & \multicolumn{3}{|c|}{ Período II } \\
\hline & Botón floral & Fruto verde & Fruto maduro & Botón floral & Fruto verde & Fruto maduro \\
\hline Botón floral & $\begin{array}{c}0,8518 \\
(0,0000)\end{array}$ & - & - & $\begin{array}{c}0,8012 \\
(0,001)\end{array}$ & - & - \\
\hline Fruto verde & - & $\begin{array}{c}0,8247 \\
(0,0000)\end{array}$ & - & - & $\begin{array}{c}0,9101 \\
(0,0000)\end{array}$ & - \\
\hline Fruto maduro & - & - & $\begin{array}{c}0,9484 \\
(0,0000)\end{array}$ & - & - & $\begin{array}{c}0,8783 \\
(0,0000)\end{array}$ \\
\hline
\end{tabular}

Tabla 2. Duración media de las estructuras reproductivas individuales de $O$. bataua.

Table 2. Average length of individual reproductive structures of $O$. bataua.

\begin{tabular}{|c|c|c|c|c|c|c|c|c|}
\hline \multirow{2}{*}{$\begin{array}{c}\text { Estructura } \\
\text { reproductiva }\end{array}$} & \multirow{2}{*}{$\begin{array}{c}\text { Duración } \\
\text { (meses) }\end{array}$} & \multirow[t]{2}{*}{ DE } & \multirow{2}{*}{$\begin{array}{l}\text { CV } \\
(\%)\end{array}$} & \multicolumn{2}{|c|}{ IC $(95 \%)$} & \multirow[t]{2}{*}{$\max$} & \multirow[t]{2}{*}{$\min$} & \multirow[t]{2}{*}{$\mathbf{n}$} \\
\hline & & & & $\mathbf{L S}$ & LI & & & \\
\hline Botón floral & 6,27 & 2,52 & 40,20 & 6,57 & 5,96 & 17,50 & 2,30 & 249 \\
\hline Flor & 0,80 & 0,35 & 44,02 & 0,92 & 0,69 & 1,50 & 0,48 & 39 \\
\hline Fruto verde & 11,56 & 2,94 & 25,44 & 12,09 & 11,04 & 16,60 & 3,30 & 121 \\
\hline Fruto maduro & 5,18 & 1,75 & 33,75 & 5,60 & 4,76 & 9,82 & 2,50 & 70 \\
\hline Total & 23,81 & - & - & - & - & - & - & - \\
\hline
\end{tabular}



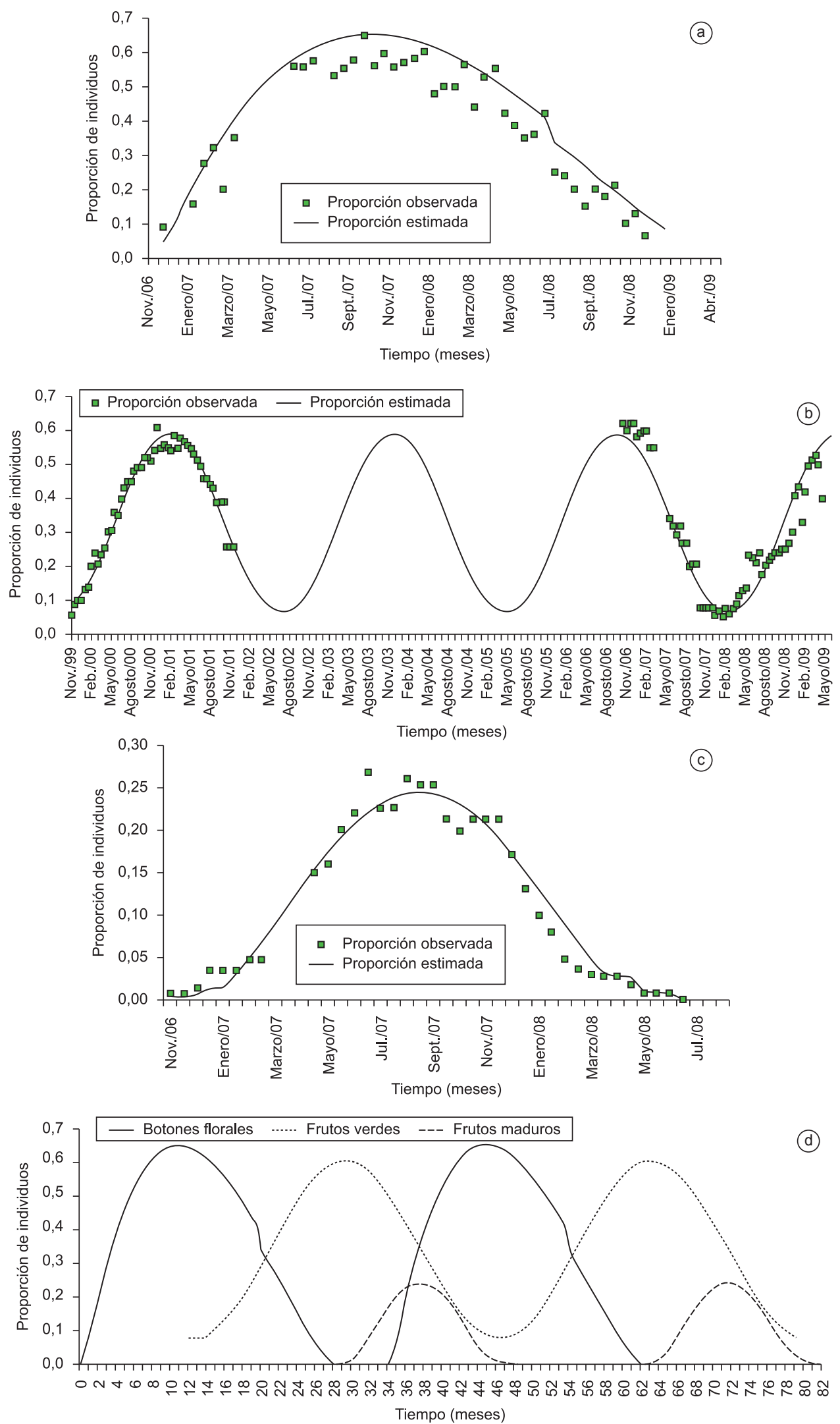

Figura 1. Fenología reproductiva de $O$. batuaua. a) Proporción de individuos de $O$. bataua que presenta la fenofase botón floral; b) Proporción de individuos de $O$. bataua que presenta la fenofase fruto verde y simulación de su patrón de ocurrencia mediante un modelo sinusoidal; c) Proporción de individuos de $O$. bataua que presentan la fenofase fruto maduro; d) Modelación del ciclo fenológico de $O$. bataua en el Milpesal Puro.

Figure 1. Reproductive phenology of $O$. batuaua. a) Observed and estimated values for proportion of individuals of $O$. bataua showing flower buds; b) Observed and estimated values for proportion of individuals of $O$. bataua showing green fruits; c) Observed and estimated values for proportion of individuals of $O$. bataua showing ripe fruits; d) Modelling of the phenological cycle of $O$. bataua in Pure Milpesal. 
MM, 75\% de los botones florales alcanzó la fase fruto verde y $15 \%$ llegó a fruto maduro ( $25 \%$ de frutos verdes). O. bataua presentó una densidad poblacional de 278 palmas.ha $^{-1}$ en MP, de las cuales $47 \%$ produjo frutos maduros (131 palmas.ha ${ }^{-1}$ ): $27 \%$ produjo un racimo, $17 \%$ produjo dos racimos y $3 \%$ produjo tres racimos. En MM, la especie está representada por 82 palmas.ha ${ }^{1}$, de las cuales $26 \%$ produjo frutos maduros (21,3 palmas.ha- $\left.{ }^{-1}\right): 20 \%$ produjo un racimo y $6 \%$ produjo dos racimos.

La producción de frutos maduros ocurrió cada 34 meses, con un estimado de 192 racimos ha ${ }^{-1}$ en MP y 21 racimos.ha ${ }^{-1}$ en MM. El peso medio de un racimo de frutos maduros fue $15,7 \mathrm{~kg}(\mathrm{DE}=6,02)$, con un promedio de 1255 frutos por racimo $(\mathrm{DE}=515,46)$. De esta forma, se proyectó una producción de $3014 \mathrm{~kg}$ (DE: 1156,5) y $330 \mathrm{~kg}$ (DE: 126,5) de frutos maduros.ha-1 (por ciclo productivo) en MP y MM, respectivamente. La productividad en MP fue 9,1 veces superior a la de MM; sin embargo, el número de palmas presentes en MP fue sólo 3,4 veces superior, lo que indica una mayor productividad individual en el ecosistema donde $O$. bataua es dominante.

\section{Discusión}

\section{Ciclo reproductivo de la población}

El grado de sincronía de los eventos reproductivos de $O$. bataua es muy variable; se reportan valores asincrónicos de $25 \%$ en el Pacífico colombiano (Collazos \& Mejía 1988), hasta de alta sincronía en la Amazonía colombiana, donde alcanzó 90\% (Vélez 1992). Nuestros

Tabla 3. Duración media de las estructuras reproductivas individuales entre ecosistemas en cada período de registro.

Table 3. Average length of individual reproductive structures between ecosystems in each period recorded.

\begin{tabular}{clrrrrr}
\hline \multirow{2}{*}{ Período } & Estructura & \multicolumn{2}{c}{ Duración (meses) } & & \multicolumn{2}{c}{ ANOVA } \\
\cline { 3 - 4 } \cline { 6 - 7 } & reproductiva & MM & MP & & $\boldsymbol{F}$ & Valor- $\boldsymbol{p}$ \\
\hline I & Botón floral & 5,62 & 6,31 & & 2,82 & 0,094 \\
& Flor & 0,55 & 0,73 & & 3,15 & 0,082 \\
& Fruto verde & 10,63 & 12,75 & & 2,59 & 0,110 \\
II & Botón floral & 4,32 & 5,15 & & 0,53 & 0,471 \\
& Flor & 0,73 & 0,82 & & 0,29 & 0,594 \\
& Fruto verde & 10,09 & 10,96 & & 0,42 & 0,524 \\
& Fruto maduro & 4,16 & 5,29 & & 2,72 & 0,104 \\
\hline
\end{tabular}

Tabla 4. Correlación entre precipitación y fenofases de O. bataua (valor superior: coeficiente de correlación de Pearson, valor inferior entre paréntesis: significancia estadística).

Table 4. Correlation between rainfall and phenophases of $O$. bataua (top of each cell: Pearson's correlation coefficient, bottom in parenthesis: $\mathrm{P}$ value).

\begin{tabular}{lccc}
\hline \multicolumn{1}{c}{ Precipitación } & \multicolumn{3}{c}{ Fenofases } \\
\cline { 2 - 4 } & $\begin{array}{c}\text { Botón } \\
\text { floral }\end{array}$ & $\begin{array}{c}\text { Frutos } \\
\text { verdes }\end{array}$ & $\begin{array}{c}\text { Frutos } \\
\text { maduros }\end{array}$ \\
\hline Mes corriente & $-0,0745$ & $-0,1163$ & 0,0034 \\
$(\mathrm{~N}=54)$ & $(0,5926)$ & $(0,4021)$ & $(0,9808)$ \\
Retraso temporal - 1 mes & $-0,0105$ & $-0,1075$ & 0,0737 \\
$(\mathrm{~N}=54)$ & $(0,9398)$ & $(0,4393)$ & $(0,5966)$ \\
Retraso temporal - 2 meses & $-0,0048$ & $-0,0579$ & $-0,0083$ \\
$(\mathrm{~N}=53)$ & $(0,9727)$ & $(0,6803)$ & $(0,9527)$ \\
Retraso temporal - 3 meses & $-0,1917$ & $-0,0579$ & $-0,0212$ \\
$(\mathrm{~N}=52)$ & $(0,1734)$ & $(0,6836)$ & $(0,8815)$ \\
Retraso temporal - 4 meses & $-0,1438$ & $-0,0584$ & $-0,0857$ \\
$(\mathrm{~N}=51)$ & $(0,3192)$ & $(0,6872)$ & $(0,5540)$ \\
\hline
\end{tabular}

resultados indican que, en general, la sincronía a nivel poblacional fue baja, con picos de 62 y $27 \%$ para las fenofases fruto verde y fruto maduro, respectivamente. Estos registros son similares a los hallados por Rojas-Robles \& Stiles (2009) en los Andes colombianos, quienes encontraron valores máximos de sincronía de $70 \%$ para frutos verdes y $30 \%$ para frutos maduros.

De acuerdo con la clasificación propuesta por Newstrom et al. (1994), la ocurrencia de botones florales y frutos verdes presentó un patrón continuo y cíclico, ya que estos eventos siempre se observaron durante el tiempo de estudio, con períodos de mayor y menor producción claramente diferenciados. Por su parte, la producción de frutos maduros no fue continua, con picos cada 34 meses. El patrón continuo de la ocurrencia de frutos verdes y su comportamiento cíclico, detectado a partir de la modelación en un período de 9 años (Figura 1b) y el cual se corroboró para las fenofases botones florales y frutos maduros, sugiere que la fenología reproductiva de $O$. bataua a nivel de población, en la región del Medio Atrato, sigue un patrón continuo y cíclico a lo largo del tiempo, con una duración aproximada de 4 años desde la aparición del botón floral hasta la maduración y caída de los frutos (Figura 1d). Este patrón continuo también ha sido reportado por otros autores como Henderson et al. (2000) en la Amazonía brasileña y Stevenson et al. (2008) en la Amazonía occidental de Colombia, el cual responde probablemente a la baja sincronía en los eventos reproductivos a nivel individual.

Igualmente, al comparar nuestros resultados con los de los Andes (Rojas-Robles \& Stiles 2009), encontramos gran similitud en la duración del ciclo reproductivo poblacional, ya que estos autores determinaron que el ciclo poblacional tardó cerca de 4 años. Estos resultados están en contraposición con lo que esperábamos, lo cual es que los patrones fenológicos son diferentes entre regiones geográficas distintas y en un rango amplio de condiciones ambientales. Los bosques de los Andes, donde se realizó el estudio de Rojas-Robles \& Stiles (2009), se encuentran sobre suelos de tierra firme, en condiciones topográficas de montaña, a una altitud superior a $1000 \mathrm{msnm}$, con temperatura promedia anual de $23{ }^{\circ} \mathrm{C}$ y precipitación anual inferior a $2000 \mathrm{~mm}$; estas condiciones son muy diferentes a las de los bosques del Chocó del presente estudio: bosques sobre suelos aluviales de la llanura de inundación del Río Atrato (Cortés 1993), topografía plana, altitud menor de $100 \mathrm{msnm}$ (Galvis \& Mojica 1993), temperatura promedia de $28{ }^{\circ} \mathrm{C}$ y precipitación anual de $6000 \mathrm{~mm}$ aproximadamente, sin meses ecológicamente secos (Eslava 1993).

Por su parte, las diferencias encontradas en el comportamiento fenológico y en la productividad de la especie respecto a otros estudios (Collazos \& Mejía 1988, Vélez 1992, Miller 2002) puede deberse a los tiempos de evaluación, ya que la duración de tales estudios (menos de 3 años) pudo limitar la clara identificación del ciclo fenológico. De acuerdo con Newstrom et al. (1994), puesto que muchas especies presentan ciclos fenológicos multianuales, se requieren períodos de observación prolongados, por lo menos de 5 años, para describir completamente estos patrones.

El bajo número de inflorescencias fecundadas utilizado en el cálculo del tiempo necesario para su desarrollo, se debió a la corta duración de la fenofase flor; la antesis ocurrió, en la mayoría de los casos, entre dos muestreos sucesivos, pasando de botón floral a racimo verde entre observaciones consecutivas.

\section{Relación fenología - clima}

La floración, fructificación y crecimiento de muchas especies de plantas tropicales ocurren de forma episódica, y sus picos se han relacionado con variables ambientales y factores como la disponibilidad de agua y de luz (Borchert 1983, 1994, Borchert \& Rivera 2001, Stevenson et al. 2008). Por su parte, la fenología de $O$. bataua se ha relacionado con variables climáticas (Collazos 
\& Mejía 1988, Miller 2002, Ruiz \& Alencar 2004, Rojas-Robles \& Stiles 2009); sin embargo, los resultados de estos estudios no permiten apreciar una relación clara entre las variables. De acuerdo con Williams-Linera (2003), la fenología de muchas especies no responde igualmente a todas las variables ambientales e, incluso, puede no existir relación alguna entre el clima y su fenología.

Cuando evaluamos la relación entre la producción de flores, frutos verdes y frutos maduros de $O$. bataua con la precipitación, no encontramos una correlación significativa. Igualmente, debido a la estrecha relación entre precipitación y nivel del río, los resultados sugieren que este último y la inundabilidad no determinan el comportamiento de la fenología reproductiva de la especie. Borchert (1983) y Ashton et al. (1988) plantearon que el grado de sincronía de los eventos fenológicos puede indicar la fuente de control, dado que respuestas fenológicas asincrónicas son gobernadas probablemente por factores endógenos, mientras que respuestas más concentradas en el tiempo son originadas por estímulos climáticos. De este modo, la alta variabilidad de las relaciones clima-fenofases encontrada en diversos estudios de esta especie, la baja sincronía predominante en cada fenofase, la larga duración del período reproductivo y la similitud en la ocurrencia de eventos fenológicos entre regiones geográficas con patrones climáticos diferentes (e.g. Andes-Chocó Biogeográfico), sugieren que el comportamiento cíclico de la fenología reproductiva de $O$. batau responde a factores endógenos y/o a relaciones de tipo ecológico-evolutivo. En efecto, a pesar de que el estudio fenológico de esta especie realizado en los Andes encontró correlaciones significativas entre algunas variables climáticas y fenofases (RojasRobles \& Stiles 2009), los autores concluyeron que las diferencias interanuales en las variables climáticas son insuficientes para explicar el ciclo multianual de O. bataua. Asimismo, reconocen que sus resultados son incompatibles con la hipótesis de causación directa de los eventos fenológicos por las condiciones ambientales.

\section{Productividad}

El número estimado de palmas reproductivas por unidad de área en los bosques del Medio Atrato fue superior a lo reportado por Miller (2002), en la Amazonía, y por Collazos \& Mejía (1988), en el Pacífico colombiano. La densidad poblacional de individuos reproductivos en MP fue incluso superior a la reportada por Peters et al. (1989) en los bosques oligárquicos estacionalmente inundables de la Amazonía peruana, siendo MP la asociación vegetal más homogénea (referida a $O$. bataua) reportada hasta el momento.

La falta de polinización de muchas flores recién expuestas, así como el secamiento de numerosos racimos de frutos verdes durante su desarrollo, llevó a que un porcentaje relativamente bajo de estructuras lograran convertirse en frutos maduros. En contadas ocasiones se observaron ataques de insectos a los botones florales, situación contraria a la reportada por Collazos \& Mejía (1988), quienes identificaron a Rhynchophorus palmarum (Curculionidae) como el agente que destruyó un número importante de botones florales. De otro lado, Balick (1992) planteó que el desarrollo de las inflorescencias y frutos puede abortarse por el estado desfavorable de recursos nutritivos foto-asimilables.

El peso y tamaño de los racimos de frutos maduros fueron variables; los valores aquí determinados $(15,7 \mathrm{~kg})$ fueron intermedios entre los reportados por diversos autores $(6,2-22 \mathrm{~kg}$ ) (Collazos \& Mejía 1988, Balick 1992, Vélez 1992). Aunque la producción de frutos maduros, tanto en MP como en MM, ocurrió durante la misma época, la productividad individual en MP fue mayor. Se ha sugerido que la reducción de la productividad de los bosques se debe principalmente a una disminución en la cantidad de radiación fotosintéticamente activa absorbida por las copas debido a la competencia por luz, la cual está asociada a competencia por nutrientes en el suelo (Kimmins
1997). Este efecto combinado probablemente sea la causa de la menor productividad de los individuos de $O$. bataua en $\mathrm{MM}$, los cuales no alcanzan la parte superior del dosel y ocurren en densidades muy inferiores a las que se presentan en MP, por lo que posiblemente la cantidad de radiación fotosintéticamente activa que reciben es menor debido a la competencia de las demás especies en estos bosques.

En consonancia con lo anterior, Schaefer \& Schmidt (2002) reportaron una mayor productividad de frutos en los individuos del dosel del bosque; por esto, la mayor productividad encontrada en MP puede deberse a que las palmas dominan el dosel en este ecosistema, reciben luz plena y están sometidas a baja competencia por este recurso. Si esta hipótesis fuera correcta, el manejo de los bosques de MM, con el objeto de producir frutos cosechables de esta especie, debería incluir tratamientos controlados de apertura de dosel, con bajo impacto, dirigidos a aumentar la radiación incidente sobre las palmas.

Bajo otra óptica, los períodos de inundación más prolongados que se presentan en MM pueden reducir la productividad de frutos, ya que $O$. bataua, a diferencia de otras especies de palmas como E. oleracea, adaptadas a ambientes inundables, no presenta neumatóforos, los cuales son un mecanismo que facilita el movimiento de oxígeno entre raíces, hojas y tallos (Kimmins 1997). Los suelos inundables se vuelven hipóxicos rápidamente $\mathrm{y}$, en consecuencia, limitan la disponibilidad de oxígeno para las raíces; esto frena las tasas de productividad y fotosíntesis, e inhibe la síntesis de etileno, indispensable para la maduración de los frutos (Salisbury \& Ross 1992).

Finalmente, dado el comportamiento descrito de la fenología reproductiva de $O$. bataua, se espera que haya un pico de producción de frutos maduros cada 34 meses; sin embargo, habrá un período donde la oferta del recurso es nula o muy baja. Las altas pérdidas que ocurren desde la fenofase botón floral hacia fruto verde y fruto maduro, dan lugar a un amplio campo de investigación y manejo, lo cual eventualmente permitiría aumentar la producción de frutos maduros para proyectos basados en su explotación sostenible. Lograr este propósito requiere más estudios sobre la especie a nivel individual, particularmente sobre los agentes que causan los abortos de inflorescencias y la no maduración de los frutos verdes.

\section{Aspectos de conservación y manejo}

Las comunidades humanas asentadas en la región viven en condiciones de extrema pobreza, sufren problemas de salud debido a la dieta deficiente y prácticamente no desarrollan labores productivas comerciales. El modelo extractivista utilizado para satisfacer sus necesidades ha generado sobre-explotación y disminución de los recursos que tienen mayor valor en los mercados, particularmente la madera (fuente de ingresos más importante), lo que ha puesto en peligro de extinción a algunas especies de fauna y flora (Díez 1996).

O. bataua constituye uno de los principales recursos no maderables de la región y, aunque los habitantes reconocen sus beneficios, sólo la utilizan ocasionalmente para aprovechar sus frutos maduros, los cuales cosechan al talar la palma; no obstante, esta práctica impide el uso futuro del recurso. En consecuencia, el manejo sostenible de los frutos implica formas de aprovechamiento diferentes, como escalar la palma o cosechar el racimo desde el piso con el uso de ganchos, como el de tipo malayo usado en este estudio. Este último método es apropiado y más seguro que el escalado de las palmas, puesto que no todos los pobladores están adiestrados para subir a ellas; además, el transporte y uso del gancho no ofrece dificultades dentro del bosque. De esta forma, mediante aprovechamientos no destructivos y con intensidades que no impacten significativamente el reclutamiento de la especie, así como a las poblaciones animales que la incluyen en su 
dieta, principalmente frugívoros (Aguilar 2006, Núñez-Avellaneda \& Rojas-Robles 2008), podrán darse cosechas periódicas que provean ingresos a las comunidades locales y, al mismo tiempo, protejan la integridad del bosque.

Desde los puntos de vista ecológico y económico, la utilización sostenible de estos bosques puede ser una alternativa viable. La dominancia natural de $O$. bataua en los ecosistemas de esta región facilita su manejo y aprovechamiento, lo cual puede lograrse con baja inversión de capital y mano de obra, y sin la aplicación de fertilizantes o pesticidas. Estas características pueden considerarse favorables si se tienen en cuenta las condiciones socio-económicas de las comunidades locales. No obstante, el éxito futuro de esta alternativa productiva requiere que exista un mercado donde el aprovechamiento de los frutos sea una mejor opción que la explotación de los recursos maderables. Entender los factores que controlan las decisiones de manejo y los efectos ecológicos de éste (Weinstein \& Moegenburg 2004), son condiciones necesarias para que los bosques puedan proveer una opción productiva de largo plazo para las poblaciones allí asentadas, donde se integre el desarrollo económico y social con la conservación de las coberturas forestales.

\section{Conclusiones}

El comportamiento de la fenología de O. bataua es continuo y cíclico, por tanto, predecible, con una duración aproximada de 4 años. La baja sincronía y la temporalidad del ciclo reproductivo de la especie obedecen, probablemente, a que no existe una relación entre precipitación y fenología que permita explicar su patrón; alternativamente, su comportamiento recurrente posiblemente responde a factores endógenos o a relaciones de tipo ecológicoevolutivo. Para implementar programas de manejo y aprovechamiento de la especie, es importante considerar que la producción de frutos maduros no es continua, sino que se presenta durante 22 meses, alternada con 12 meses de oferta nula. La cosecha puede alcanzar valores de 3014 y $330 \mathrm{~kg} \cdot \mathrm{ha}^{-1}$ en MP y MM, respectivamente, por período productivo. El manejo sostenible de estos bosques, a través del aprovechamiento periódico de frutos de O. bataua, puede generar alimento e ingresos económicos a las comunidades locales y mejorar sus condiciones de vida; asimismo, puede promover la conservación de los ecosistemas.

\section{Agradecimientos}

Agradecemos al Departamento Administrativo de Ciencia, Tecnología e Innovación - Colciencias, por su apoyo a través del programa “Jóvenes Investigadores e Innovadores 2007” y del contrato 404/1998: "Fundamentos biológicos para el aprovechamiento sostenible de productos de las especies Euterpe oleracea y Oenocarpus bataua en la región del Medio Atrato, Colombia"; a la Dirección Nacional de Investigación de la Universidad Nacional de Colombia, por la financiación del proyecto 30803997: "Estructura y dinámica de bosques inundables en el Pacífico colombiano"; a CORPOURABÁ, subregional Atrato, por el apoyo logístico prestado en campo; y a los Concejos Comunitarios de Villanueva y San Martín (Municipio de Vigía del Fuerte, Antioquia).

\section{Referencia}

AGUILAR, Z. 2006. Influencia de los indígenas Huaorani en la conservación de Oenocarpus bataua, Arecaceae, en el Parque Nacional y Reserva de Biosfera Yasuní, Amazonía ecuatoriana. Lyonia 10(2):83-90.

ASHTON, P., GIVNISH, T.J. \& APPANAH, S. 1988. Staggered flowering in the Dipterocarpaceae: new insights into floral induction and the evolution of mast fruiting in the aseasonal tropics. Am. Nat. 132:44-66.
BACH, C.S. 2002. Phenological patterns in monsoon rainforests in the Northern Territory, Australia. Austral Ecol. 27:477-489.

BALICK, M.J. 1992. Jessenia y Oenocarpus: palmas aceiteras neotropicales dignas de ser domesticadas. Estudio FAO: Producción y Protección Vegetal, FAO, Roma.

BENCKE, C. \& MORELLATO, P. 2002a. Estudo comparativo da fenologia de nove espécies arbóreas em três tipos de floresta atlântica no Sudeste do Brasil. Rev. Brasil. Bot. 25(2):237-248.

BENCKE, C. \& MORELLATO, P. 2002b. Comparação de dois métodos de avaliação da fenologia de plantas, sua interpretação representação. Rev. Brasil. Bot. 25(3):269-275.

BORCHERT, R. \& RIVERA, G. 2001. Photoperiodic control of seasonal development and dormancy in tropical stem succulent trees. Tree Physiol. $21: 213-221$

BORCHERT, R. 1983. Phenology and control flowering in tropical trees. Biotropica 15:81-89.

BORCHERT, R. 1994. Soil and stem water storage determine phenology and distribution of tropical dry forest trees. Ecology 75:1437-1449.

BORCHERT, R. 1999. Climatic periodicity, phenology, and cambium activity in tropical dry forest trees. IAWA J. 20(3):239-247.

BORCHERT, R., MEYER, S.A., FELGER, R.S. \& PORTER-BOLLAND, L. 2004. Environmental control of flowering periodicity in Costarican and Mexican tropical dry forest. Global Ecol. Biogeogr. 13:409-425.

COLLAZOS, M.E. \& MEJÍA, M. 1988. Fenología y poscosecha de milpesos Jessenia bataua (Mart.) Burret. Acta Agron. 38:53-63.

CORTES, A. 1993. Los suelos. In Colombia Pacífico. (P. Leyva, ed.). Tomo 1. Fondo para la Protección del Medio Ambiente "José Celestino Mutis" (FEN Colombia), Bogotá, p.148-155.

DÍEZ, M.C. 1996. Las unidades familiares de producción en el municipio de Vigía del Fuerte (Ant.). Universidad Nacional de Colombia, Medellín.

DO, F.C., GOUDIABY, V.A., GIMENEZ, O., DIAGNE, A.L., DIOUF, M., ROCHETEAU, A. \& AKPO, L.E. 2005. Environmental influence on canopy phenology in the dry tropics. Forest Ecol. Manag. 215:319328.

ESLAVA, J.A. 1993. Climatología. In Colombia Pacífico. (P. Leyva, ed.). Tomo 1. Fondo para la Protección del Medio Ambiente "José Celestino Mutis" (FEN Colombia), Bogotá, p.148-155.

GALEANO, G. \& BERNAL, R. 1987. Palmas del departamento de Antioquia: región occidental. Universidad Nacional de Colombia, Bogotá.

GALVIS, J. \& MOJICA, J. 1993. Geología. In Colombia Pacífico (P. Leyva, ed.). Tomo 1. Fondo para la Protección del Medio Ambiente "José Celestino Mutis" (FEN Colombia), Bogotá, p.148-155.

HAUGAASEN, T. \& PERES, C.A. 2005. Tree phenology in adjacent Amazonian flooded and unflooded forests. Biotropica 37(4):620-630.

HENDERSON, A., GALEANO, G. \& BERNAL, R. 1995. Field guide to the palms of the Americas. Princeton University, New Jersey.

HENDERSON, G., FISCHER, B., SCARIOT, A., WHITAKER, M.A. \& PARDINI, R. 2000. Flowering phenology of a palm community in a Central Amazon forest. Brittonia 52(2):149-159.

HOLDRIDGE, L. R. 1978. Ecología basada en zonas de vida. IICA, San José.

KAHN, F. 1991. Palms as key swamp forest resources in Amazonia. Forest Ecol. Manag. 38:133-142.

KIMMINS, J.P. 1997. Forest ecology: a foundation for sustainable management. $2^{\text {nd }}$ ed. Prentice Hall, University of British Columbia, New Jersey.

McLAREN, K.P. \& McDONALD, M.A. 2005. Seasonal patterns of flowering and fruiting in a dry tropical forest in Jamaica. Biotropica 37(4):584-590.

MILLER, C. 2002. Fruit production of the ungurahua palm (Oenocarpus bataua subsp. bataua, Arecaceae) in an indigenous managed reserve. Econ. Bot. 56:165-176. 
MORCOTE-RÍOS, G., CABRERA-BECERRA, G., MAHECHA-RUBIO, D., FRANKY-CALVO, C.E. \& CAVELIER I.F. 1998. Las palmas entre los grupos cazadores-recolectores de la Amazonía colombiana. Caldasia 20:57-74.

NEWSTROM, L.E., FRANKIE, G.W. \& BAKER, H.G. 1994. A new classification for plant phenology based on flowering patterns in lowland tropical rain forest trees at La Selva, Costa Rica. Biotropica 26(2):141-159.

NÚÑEZ-AVELLANEDA, L. \& ROJAS-ROBLES. R. 2008. Biología reproductiva y ecología de la polinización de la palma milpesos Oenocarpus bataua en los Andes colombianos. Caldasia 30:101-125.

PETERS, C.M., BALICK, M.J., KAHN, F. \& ANDERSON, A.B. 1989. Oligarchic forest of economy plants in Amazonia: Utilization and conservation of an important tropical resource. Conserv. Biol. 3(4):341-349.

ROJAS-ROBLES, R. \& STILES, G. 2009. Analysis of supra-annual cycle: reproductive phenology of the palm Oenocarpus bataua in a forest of the Colombian Andes. J. Trop. Ecol. 25:41-51.

RUIZ, R.R. \& ALENCAR, J.C. 2004. Comportamento fenológico da palmeira patauá (Oenocarpus bataua) na reserva florestal Adolpho Ducke, Manaus, Amazonas, Brasil. Acta Amaz. 34:553-558.

SALISBURY, F.B. \& ROSS, C.W. 1992. Plant physiology. $4^{\text {th }}$ ed. Wadsworth, California.

SCHAEFER, H.M. \& SCHMIDT, V. 2002. Vertical stratification and caloric content of the standing fruit crop in a tropical lowland forest. Biotropica 34(2):244-253
SCHÖNGART, J., PIEDADE, M.T., LUDWIGSHAUSEN, S., HORNA, V. \& WORBES, M. 2002. Phenology and stem-growth periodicity of tree species in Amazonian flood plain forest. J. Trop. Ecol. 18:581-597.

STEVENSON, P.R. 2004. Phenological patterns of woody vegetation at Tongue Park, Colombia: Methodological comparisons with emphasis on fruit production. Caldasia 26(1):125-150.

STEVENSON, P.R., CASTELlANOS, M.C., CORTÉS, A.I. \& LINK, A. 2008. Flowering patterns in a seasonal tropical lowland forest in Western Amazonia. Biotropica 40(5):559-567.

UNIVERSIDAD NACIONAL DE COLOMBIA. 1996. Diagnostico de las existencias de la palma Murrapo o Naidí (Euterpe oleracea M.) y del componente arbóreo en un área del municipio de Vigía de Fuerte, región del Medio Atrato, Colombia. Universidad Nacional de Colombia, Medellín.

VÉLEZ, G. 1992. Estudio fenológico de diecinueve frutales silvestres utilizados por las comunidades indígenas de la región de Araracuara Amazonía colombiana. Colomb. Amaz. 6:135-186.

WALLACE, R.B. \& PAINTER, R.L.E. 2002. Phenological patterns in a Southern Amazonian tropical forest: implications for sustainable management. Forest Ecol. Manag. 160:19-33.

WEINSTEIN, S. \& MOEGENBURG, S. 2004. Açaí palm management in the Amazon estuary: course for conservation or passage to plantations? Conservat. Soc. 2(2):316-346.

WILLIAMS-LINERA, G. 2003. Temporal and spatial phenological variation of understory shrubs in a tropical mountain cloud forest. Biotropica 35(1):28-36. 
